\title{
Redes de Sensores com Codificação BCH Distribuída
}

\author{
Eline Alves Santos, Francisco Marcos de Assis e Edmar Candeia Gurjão
}

\begin{abstract}
Resumo-O objeto deste artigo é o projeto de sistemas de classificação distribuída para redes de sensores em que cada uma de $M$ hipóteses é associada a uma palavra código de um código não linear de bloco binário de comprimento $N$. Os códigos de bloco não lineares propostos na literatura são construídos por busca aleatória sem qualquer estrutura a priori. A regra de decisão abrupta por distância mínima é utilizada no centro decisor. A abordagem apresentada neste trabalho consiste na restrição do espaço de busca às palavras código de um código BCH de comprimento conveniente por meio de um algoritmo do tipo genético que faz uso de propriedades dos códigos $\mathrm{BCH}$ para facilitar a construção do subcódigo não-linear e permitir a utilização adaptada de algoritmos de decodificação algébricos. Esta característica é potencialmente interessante nos casos em que o número de hipóteses for elevado. Resultados numéricos obtidos indicam que os resultados obtidos são similares aos obtidos com a busca sem restrição.
\end{abstract}

Palavras-Chave-Classificação distribuída, redes de sensores sem fio, codificação.

Abstract-This article is concerned to the project of distributed classification fusion schemes for wireless sensor networks where each one of $M$ hypoteses is assigned to one codeword from a binary nonlinear block code of length $N$. The nonlinear block codes proposed in the literature are built by random search without any a priori structure. The minimum distance decision rule is utilized in the fusion center. The approach presented in this work comprises in constraining the search space to that formed by the codewords from a BCH code with convenient blocklength by means utilization a kind of genetic algorithm that put into action properties of the BCH codes in order to easy building the nonlinear subcode and allowing to employ adapted algebraic decoding algorithms. This characteristic is potentially interesting if a huge number of hypotheses are presented. Numerical results obtained indicated similar performance in comparison with that obtained using codes built without any constraints.

Keywords - Distributed classification, wireless sensor networks, coding.

\section{InTRODUÇÃo}

Avanços tecnológicos nas áreas de Comunicação sem Fio e Eletrônica têm viabilizado o desenvolvimento de pequenos dispositivos com capacidade de sensoriamento, processamento de dados e comunicação, os chamados nós sensores ou ainda sensores "inteligentes". A produção em larga escala destes dispositivos vem barateando o seu custo, sendo possível pensar em aplicações de sensoriamento remoto com centenas ou mesmo milhares de nós sensores [1].

Eline Alves Santos, Francisco Marcos de Assis e Edmar Candeia Gurjão Departamento de Engenharia Elétrica da Universidade Federal de Campina Grande, Paraíba, Brasil, E-mails: eline.santos@ee.ufcg.edu.br, fmarcos@dee.ufcg.edu.br, ecandeia@dee.ufcg.edu.br. Este trabalho foi parcialmente financiado pelo CNPq (309431/2006.9)
O conjunto de vários nós sensores densamente distribuídos em uma região de interesse, com o objetivo de coletar, processar, armazenar e transmitir dados acerca de um ambiente, formam uma rede de sensores sem fio (RSSF). Devido ao grande leque de aplicações e desafios a serem superados, as redes de sensores têm despertado o interesse da comunidade científica.

Neste trabalho consideramos o problema da classificação de um evento ou objeto detectado, baseada nas observações de nós sensores distribuídos no ambiente, esta é uma aplicação importante das redes de sensores e por isso tem recebido bastante atenção [2], [3], [4].

Devido a restrições de energia em RSSFs é importante reduzir a comunicação, uma vez que a unidade de comunicação do nó sensor é uma das que mais consome energia ${ }^{1}$. Outra questão importante em redes de sensores é capacidade de tolerância à falhas, alguns nós podem ser danificados ou sofrer interferências do ambiente e substituí-los pode ser inviável, entretanto, isto não deve comprometer o funcionamento adequado da rede.

Em [6] e [7] propõe-se uma sistema de classificação de múltiplas hipóteses a partir de mensagens binárias enviadas pelos sensores, em que a tolerância a falhas é conseguida com a utilização de códigos corretores de erro. A ideia consiste em relacionar univocamente palavras códigos distintas a hipóteses distintas. Cada sensor responde ao fenômeno observado produzindo somente um bit que é transmitido a um centro decisor por um canal de comunicações sujeito a ruído aditivo gaussiano branco (AWGN). Admite-se que possam ocorrer erros na entrada dos sensores. O conjunto dos $\mathrm{N}$ bits nas saídas dos nós sensores é interpretado como uma palavra código correspondente a uma das M hipóteses, possivelmente contendo alguns erros. A palavra recebida é codificada e a saída do centro de fusão é a estimativa da hipótese observada pelo conjunto dos sensores. A regra de decisão utilizada no centro de fusão é distância de Hamming mínima, decide-se pela hipótese associada à palavra do código com a menor distância da palavra recebida.

$\mathrm{Na}$ abordagem proposta em [6] e [7], constrói-se, utilizandose Simulated Annealing ou Cyclic Column Replacement, uma matriz $M \times N$ denominada matriz código (não confundir com a matriz geradora de um código), em que suas linhas correspondem às palavras códigos que foram associadas a cada uma das $M$ hipóteses e as colunas representam a regra de classificação empregada em cada um dos $N$ nós sensores.

\footnotetext{
${ }^{1}$ Transmitir $1 \mathrm{~Kb}$ a uma distância de 100 metros custa tanto em termos de consumo de energia quanto executar 3 milhões de instruções em um processador de propósito geral [5].
} 
Neste trabalho propõe-se uma abordagem baseada em códigos de bloco lineares, mais especificamente em códigos BCH (Bose, Chaudhuri e Hockqueguem), que são códigos amplamente utilizados e sua estrutura propicia a construção de codificadores e decodificadores eficientes. De maneira que as palavras código que formam as linhas da matriz código pertencem a um código $\mathrm{BCH}$, formando um sub-código. Este sub-código não é linear, entretanto é possível a utilização de algoritmos de decodificação algébrica, diferentemente de [6] e [7] onde os códigos utilizados não possuem nenhuma estrutura e a decodificação só pode ser feita por comparação da palavra recebida com as palavras do código.

Propõe-se também para a seleção da matriz código mais adequada a utilização de um algoritmo genético guiado por código proposto em [8], [9] que explora as propriedades dos códigos lineares.

O restante deste artigo está organizado da seguinte forma. Na seção II será detalhado o modelo do sistema adotado; a seção III abordará os limitantes que avaliam o desempenho do sistema dada uma matriz; a seção IV descreve o projeto da matriz código; na seção V são apresentados os resultados obtidos; por fim, na seção VI serão feitas algumas considerações finais.

\section{Formulação do PROBlema}

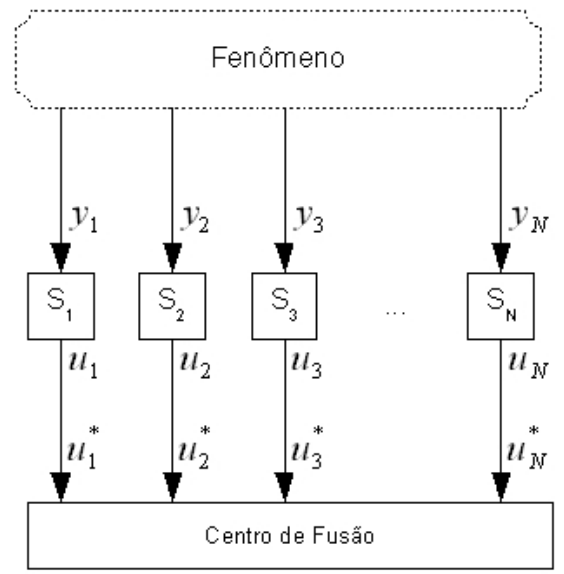

Fig. 1. Modelo do Sistema.

Considere o problema de classificação de alvos ou eventos. Seja $M$ o número de hipóteses possíveis e $N$ o número de nós sensores, todos observando o mesmo fenômeno. Assumese que os nós sensores não se comunicam entre si e que não existe realimentação do centro de fusão para os sensores locais. Este modelo é mostrado na Figura 1, ele pode servir para representar tanto a rede completa, como partes dela, e então o centro de fusão passa a ser um nó agregador de informações de determinada região da rede.

As observações dos nós sensores são representadas por $y_{j}$, em que $j=1, \ldots, N$. Admite-se que as interferências presentes nos nós sensores são estatisticamente independentes de modo que as observações $\{y\}_{j=1}^{N}$ são condicionalmente independentes dada cada hipótese.
Considera-se que as observações têm distribuição Gaussiana com média $\ell$ e variância $1 / \gamma_{o}$ dado que a hipótese $H_{\ell}$ é verdadeira, e que as hipóteses são equiprováveis. Uma hipótese $H_{i}$ será declarada verdadeira para um determinado nó sensor se:

$$
\left(y_{j}-i\right)^{2} \leq \min _{0 \leq \ell \leq M-1, \ell \neq i}\left(y_{j}-\ell\right)^{2} .
$$

A probabilidade do sensor $j$ classificar sua observação como $H_{\ell}$ dado que $H_{i}$ é a hipótese verdadeira será representada por $h_{\ell \mid i}^{(j)}$. E então:

$$
h_{k \mid i}= \begin{cases}\rho_{k-i}, & k=0 \\ \rho_{k-i}-\rho_{k-i-1}, & 1 \leq k \leq M-2 \\ 1-\rho_{k-i-1}, & k=M-1\end{cases}
$$

em que $\rho_{k}=\phi\left((k+0.5) \sqrt{\gamma_{o}}\right)$ e $\phi(\cdot)$ é a função de distribuição cumulativa da normal padrão.

Baseado em sua observação, o $j$-ésimo nó sensor decide entre uma das $M$ hipóteses ou classes e envia para um centro de fusão uma saída binária $u_{j}$ que corresponderá ao elemento $c_{\ell, j}$ da matriz código $\mathbf{C}$ se a hipótese $H_{\ell}$ for considerada verdadeira.

A matriz código $\mathbf{C}$ é uma matriz $M \times N$ com elementos $c_{\ell, j} \in\{0,1\}, \ell=0, \ldots, M-1$. Cada linha de $N$ bits forma uma palavra código que corresponde a uma das hipóteses $H_{\ell} \in$ $\Omega=\left\{H_{0}, \ldots, H_{M-1}\right\}$, as colunas representam a regra de classificação adotada pelo nó sensor correspondente.

Como o canal de transmissão não é perfeito, a palavra $u^{*}=\left(u_{1}^{*}, u_{2}^{*}, \ldots, u_{N}^{*}\right)$ recebida pelo centro de fusão pode ser diferente da palavra $\mathbf{u}=\left(u_{1}, u_{2}, \ldots, u_{N}\right)$ transmitida. Assuma que o evento erro no enlace de comunicação é independente para todos os canais de comunicação entre os nós sensores e o centro de fusão, e também independente das observações $\left\{y_{j}\right\}_{j=1}^{N}$ e da hipótese verdadeira $H_{i}$, e sua probabilidade $\operatorname{Pr}\left[u_{j}^{*} \neq u_{j}\right]$ é $\epsilon_{j}$.

Considere que o canal de comunicação entre os nós sensores e o centro de fusão é um canal com ruído aditivo gaussiano branco. A probabilidade de erro no enlace de comunicação $\epsilon_{j}$ é a mesma para todos os nós sensores, de tal forma que $\epsilon_{j}=\epsilon=Q\left(\sqrt{2 \gamma_{s}}\right)$ em que $\gamma_{s}$ é a razão sinal-ruído do enlace de comunicação e a função $Q(x)$ é dada por:

$$
Q(x)=\frac{1}{\sqrt{2 \pi}} \int_{x}^{\infty} e^{-t^{2} / 2} d t .
$$

A regra de fusão é baseada na distância de Hamming mínima, a decisão final será $H_{w}$ se $w=$ $\arg \min _{0 \leq \ell \leq M-1} d\left(u^{*}, c_{\ell}\right)$, em que $c_{\ell} \triangleq\left(c_{\ell, 1}, c_{\ell, 2}, \ldots, c_{\ell, N}\right)$ representa a linha de $\mathbf{C}$ correspondente a hipótese $H_{\ell}$ e $d(x, y)$ é a distância de Hamming entre $\mathbf{x}$ e $\mathbf{y}$. Caso existam mais de uma hipótese com a mesma distância mínima da palavra recebida, então uma delas é escolhida aleatoriamente.

\section{ANÁLISE DE DESEMPENHO}

Para caracterizar o sistema é importante se obter limitantes para a probabilidade de erro do mesmo para uma certa matriz código. Em [10] foi feita uma análise de desempenho quando o número de sensores é suficientemente grande. Em [7] os limitantes de probabilidade obtidos são válidos para qualquer quantidade finita de sensores. 
A seguir será exposto um limitante superior para a probabilidade média de erro do sistema que foi obtido em [7], este limitante pode ser utilizado como um critério em um algoritmo de busca para selecionar a matriz mais adequada.

Seja a probabilidade média de erro $P_{e}$ para fusão baseada em distância de Hamming definida como:

$$
P_{e} \triangleq \frac{1}{M} \sum_{i=0}^{M-1} \operatorname{Pr}\left(\text { detecção após a fusão } \neq H_{i} \mid H_{i}\right)
$$

Considere $\epsilon_{j}=\epsilon$ para $j=1, \ldots, N$, com $0 \leq \epsilon<1 / 2$, e $h_{k \mid i}^{(j)}=h_{k \mid i}$ para todos os sensores. A probabilidade média de erro $P_{e}$ pode ser limitada superiormente por:

$$
\begin{aligned}
P_{e} & \leq \frac{1}{M} \sum_{i=0}^{M-1} \sum_{0 \leq \ell \leq M-1, \ell \neq i}\left(1-(1-2 \epsilon)^{2}\right. \\
& \left.\times\left(\frac{\sum_{k=0}^{M-1} h_{k \mid i}\left[d\left(c_{i}, c_{k}\right)-d\left(c_{\ell}, c_{k}\right)\right]}{d\left(c_{\ell}, c_{i}\right)}\right)^{2}\right)^{d\left(c_{\ell}, c_{i}\right) / 2}
\end{aligned}
$$

Observe que o limitante exposto anteriormente é função de distâncias de Hamming o que simplifica sua avaliação. Os efeitos da precisão dos nós sensores são representados pelo termo $h_{k \mid i}$ e os efeitos do ruído no enlace de comunicação são representados pelo termo $\epsilon$.

Observe também que para as palavras de um código BCH as distâncias $d\left(c_{i}, c_{k}\right), d\left(c_{\ell}, c_{k}\right)$ e $d\left(c_{\ell}, c_{i}\right)$ são limitadas inferiormente pela distância mínima do código $\mathrm{BCH}$, enquanto que para um código aleatório podem ocorrer valores menores.

Na próxima secção será descrita a metodologia de projeto da matriz, em que este limitante apresentado aqui é utilizado como critério para o algoritmo de busca que seleciona a matriz código.

\section{Projeto da Matriz}

O desempenho desta abordagem está fortemente relacionado com a matriz código escolhida. Em um primeiro momento pode-se pensar que quanto maior for a distância de Hamming mínima entre as palavras códigos da matriz melhor será o desempenho do sistema e em geral é o que acontece. Mas o padrão das colunas da matriz, que está relacionado com o desempenho da classificação que os sensores realizam, também influi no desempenho do sistema como um todo, isto dificulta um projeto analítico da matriz código.

Em [7] a matriz código é selecionada através de simulated annealing e com o limitante (2), exposto na seção anterior, como função de energia, para o caso em que $\epsilon_{j}=\epsilon$ para $j=1, \ldots, N$ e $h_{k \mid i}^{(j)}=h_{k \mid i}$ para todos os sensores. Nenhuma restrição é imposta às palavras código que compõem a matriz, estas podem ser qualquer bloco de $N$ bits.

Neste trabalho, propõe-se que essas palavras sejam restritas às palavras de um código $\mathrm{BCH}$. Um código $\mathrm{BCH}(\mathrm{n}, \mathrm{k}, \mathrm{d})$ é um subespaço vetorial linear com elementos (palavras código) de comprimento $n$, com dimensão $k$ e distância de Hamming mínima $d$. Para um código $\mathrm{BCH}(\mathrm{n}, \mathrm{k}, \mathrm{d})$ binário existem $2^{k}$ palavras-códigos que podem ser combinadas em grupos de
Entradas: função-objetivo, limitante(2), matriz geradora do código, $G_{k \times n}$

Saída: matriz código ótima ou sub-ótima $C_{M \times N}$ Inicialização:

- $t \leftarrow 0$

- inicializar $p(t) \subset\{0,1\}^{M \times k} ;$ avaliar $P(t) G$;

Iteração:

Enquanto Final $=$ Falso faça

- $P^{\prime}(t) \leftarrow$ variação $P(t)$;

- avaliar $P^{\prime}(t) G$;

- $P(t+1) \leftarrow$ selecionar $P^{\prime}(t)$;

- $t \leftarrow t+1$;

Fim

Algoritmo 1: Algoritmo Genético guiado por código para busca em dentro de um código $\mathrm{BCH}$.

Entrada: função-objetivo, limitante(2)
Saída: matriz código ótima ou sub-ótima $C_{M \times N}$
Inicialização:
• $t \leftarrow 0 ;$
• inicializar $p(t) \subset\{0,1\}^{M \times N} ;$ avaliar $P(t) ;$
Iteração:
Enquanto Final = Falso faça
- $P^{\prime}(t) \leftarrow$ variação $P(t) ;$
- avaliar $P^{\prime}(t) ;$
- $P(t+1) \leftarrow$ seleção $P^{\prime}(t) ;$
- $t \leftarrow t+1 ;$
Fim

Algoritmo 2: Algoritmo Genético para buscas sem restrições.

M para formar uma matriz código $M \times N$. Em geral o número de hipóteses é menor que a quantidade de palavras código disponíveis, isto resulta em diferentes combinações possíveis para a matriz código, então é necessário selecionar a mais adequada. Em outras palavras, realizar a busca por um subcódigo dentro de um código BCH.

Ao realizar a seleção da matriz código para a abordagem proposta aqui é preciso garantir que as palavras código (linhas da matriz) pertençam ao código BCH escolhido, escolheu-se então utilizar um algoritmo genético (AG) guiado por códigos. Este algoritmo é uma modificação do algoritmo genético padrão ${ }^{2}$ proposta em [8], [9] em que a busca é realizada no espaço das sequências de informação, que são depois codificadas em palavras código e então avalia-se a solução. Isto garantirá que todas as palavras da matriz pertencerão ao código, e a estrutura do código $\mathrm{BCH}$ será utilizada na busca pela matriz código.

Tanto em um AG padrão quanto em um AG guiado por código, uma população representa um conjunto de soluções aproximadas para o problema, cada indivíduo é uma possível solução, a população é constantemente modificada pelos operadores genéticos (seleção, recombinação e mutação) até evo-

\footnotetext{
${ }^{2}$ Algoritmos Genéticos são uma classe particular de algoritmos evolutivos que usam técnicas inspiradas pela biologia evolutiva como hereditariedade, mutação, seleção natural e recombinação.
} 
luir para uma solução ótima ou sub-ótima.

No algoritmo utilizado neste trabalho os indivíduos são representados por sequências binárias de comprimento $M \times k$, correspondendo a uma matriz com $M$ linhas e $k$ colunas. A solução é o resultado desta matriz multiplicada pela matriz geradora $\mathbf{G}_{k \times n}$ de um código BCH $(n, k, d)$, ou seja, uma matriz código $M \times N$.

A seleção é realizada pelo método roleta, ou seja, a chance de um indivíduo ser escolhido para reprodução é proporcional à sua adequação em comparação com todos os indivíduos da população. A recombinação e a mutação são realizadas utilizando-se operadores de recombinação e mutação clássicos. Para a recombinação, cada par de indivíduos será recombinado com uma probabilidade fixa chamada de taxa de cruzamento definida previamente, o ponto de corte é escolhido aleatoriamente e realiza-se a troca de segmentos correspondente. Na mutação uma posição do cromossomo de um indivíduo é selecionado aleatoriamente e seu valor é alterado com uma probabilidade fixa chamada de taxa de mutação.

A função objetivo para este algoritmo é o limitante de probabilidade da seção III, não será necessário impor nenhuma restrição quanto a distância mínima, pois seu limitante inferior é previamente conhecido. $\mathrm{O}$ pseudo-código para este algoritmo genético é o algoritmo 1, neste algoritmo $P(t)$ representa uma população com um número $Q$ de indivíduos na geração $t$ e $P^{\prime}$ é gerada pelos operadores genéticos de seleção, recombinação e mutação.

Para realizar a comparação com a abordagem sem restrições às palavras código, selecionou-se matrizes códigos a partir de um de AG sem restrições às palavras códigos, isto é, as palavras código que formam a matriz podem ser qualquer sequência binária de comprimento $N$. Neste caso, os indivíduos são sequências binárias de comprimento $M \times N$ que corresponde a uma matriz com $M$ linhas e $N$ colunas. O pseudo-código para este algoritmo genético é o algoritmo 2.

É importante ressaltar que não é objetivo deste trabalho fazer uma comparação entre selecionar a matriz código utilizando simulated annealing ou algoritmo genético guiado por código, e sim entre fazer uma busca em todo o espaço de soluções e uma busca em um espaço menor, entretanto mais estruturado.

\section{Resultados}

Nesta seção serão apresentados simulações e resultados numéricos utilizados para comparar o desempenho entre a abordagem baseada no $\mathrm{BCH}$, proposta neste trabalho, e a abordagem baseada em busca aleatória.

Em todas as buscas realizadas tanto para o AG guiado por código quanto para o AG geral utilizou-se uma taxa de cruzamento de 0.6 e uma taxa de mutação de 0.05 , a condição de parada foi o número máximo de iterações.

Primeiramente consideramos um sistema com quinze sensores $(N=15)$ para classificar oito hipóteses $(M=$ 8). De acordo com metodologia descrita na seção anterior, selecionou-se uma matriz baseada no $\mathrm{BCH}(15,5,7)$ e comparou-se com uma matriz selecionada sem restrições. As matrizes foram selecionadas considerando $\gamma_{s}=0 \mathrm{~dB}$ e $\gamma_{o}=$ $6 \mathrm{~dB}$.
Foram realizadas simulações para estimar a probabilidade de erro esperada para cada uma das matrizes mantendo-se o valor de $\gamma_{s}$ em $0 \mathrm{~dB}$ e variando o valor de $\gamma_{o}$, também foi calculado numericamente os valores do limitante (2).

Vê-se na Figura 2 que a matriz código baseada no $\mathrm{BCH}(15,5,7)$ tem quase o mesmo desempenho que e a matriz obtida sem restrições para valores de $\gamma_{o}=0 \mathrm{~dB}$ a $\gamma_{o}=$ $6 \mathrm{~dB}$, e de $\gamma_{o}=6 \mathrm{~dB}$ em diante, valor para o qual as matrizes foram otimizadas, a matriz baseada no $\mathrm{BCH}$ tem um desempenho superior. Cabe ressaltar que para altos valores de $\gamma_{o}$ a probabilidade de o nó decidir pela hipótese errada é pequena, restando apenas o efeito do canal, por exemplo para $\gamma_{o}=12 \mathrm{~dB}$ e 8 hipóteses a probabilidade de o nó sensor realizar uma classificação errada é de aproximadamente 0,02 .

As curvas que representam o cálculo numérico do limitante (2) apresentam um comportamento similar ao das curvas de simulação, embora apresentem valores muito acima dos simulados, isto decorre do fato que o limitante utilizado é folgado.

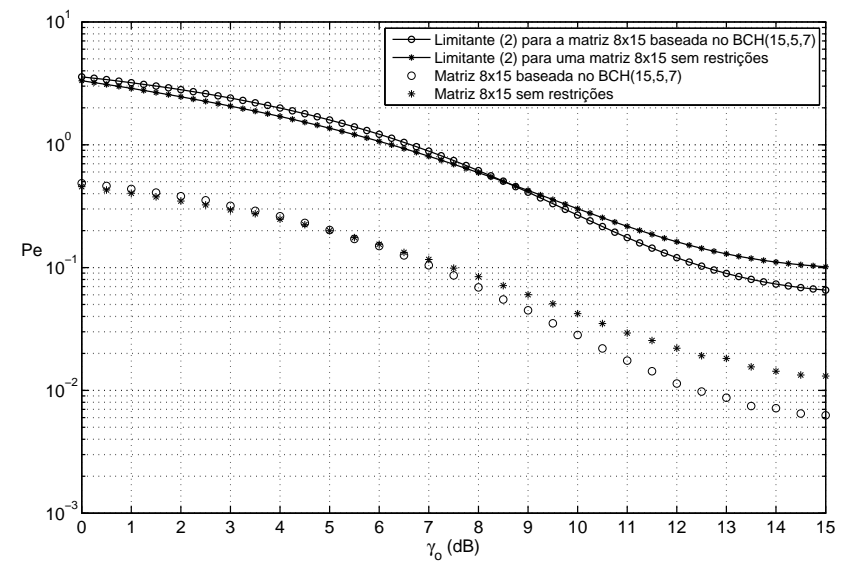

Fig. 2. Simulações e cálculo do limitante (2) para matrizes $8 \times 15$ selecionadas para $\gamma_{s}=0 \mathrm{~dB}$ e $\gamma_{o}=6 \mathrm{~dB}$. As simulações foram realizadas para $\gamma_{s}=0 \mathrm{~dB}$.

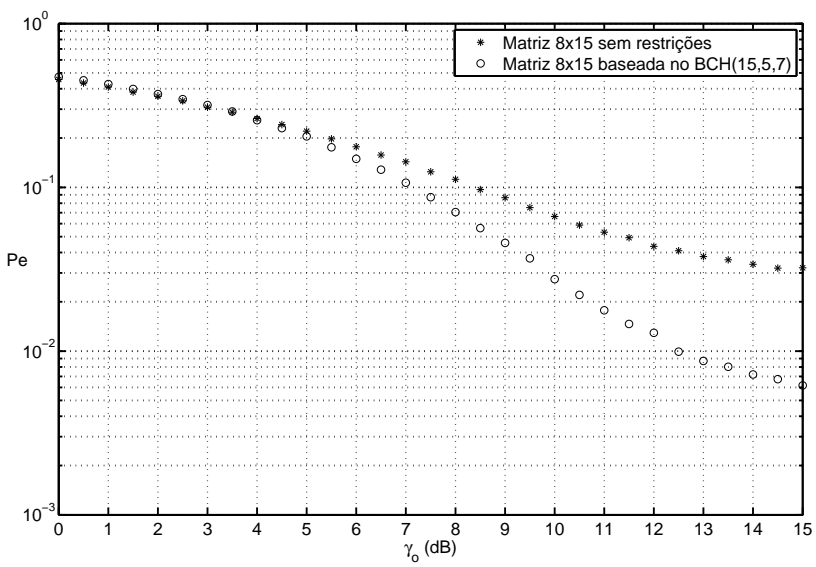

Fig. 3. Simulações para matrizes $8 \times 15$ selecionadas para $\gamma_{s}=0 \mathrm{~dB}$ e $\gamma_{o}=2 \mathrm{~dB}$. As simulações foram realizadas para $\gamma_{s}=0 \mathrm{~dB}$.

Para o mesmo sistema com quinze sensores $(N=15)$ e oito hipóteses $(M=8)$, selecionou-se matrizes para valores de $\gamma_{s}=0 \mathrm{~dB}$ e $\gamma_{o}=2 \mathrm{~dB}, \gamma_{s}=0 \mathrm{~dB}$ e $\gamma_{o}=4 \mathrm{~dB}$. Os resultados 
das simulações para estes dois casos podem ser vistos nas Figuras 3 e 4 . Novamente observa-se que as matrizes códigos baseadas no $\mathrm{BCH}(15,5,7)$ têm um desempenho ligeiramente inferior para baixos valores de $\gamma_{o}$ e que este passa a ser melhor para valores de $\gamma_{o}$ acima do valor para o qual as matrizes foram selecionadas.

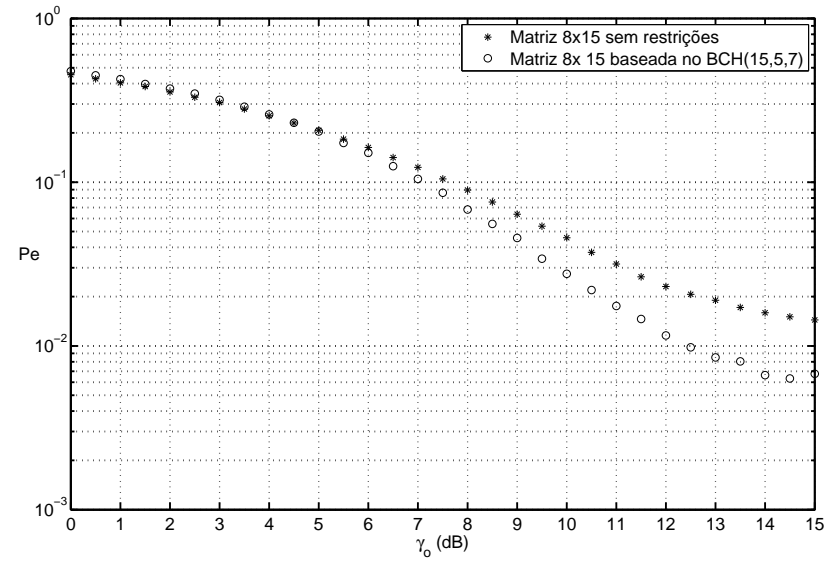

Fig. 4. Simulações para matrizes $8 \times 15$ selecionadas para $\gamma_{s}=0 \mathrm{~dB}$ e $\gamma_{o}=4 \mathrm{~dB}$. As simulações foram realizadas para $\gamma_{s}=0 \mathrm{~dB}$.

Ainda para uma rede $8 \times 15$ selecionada para valores de $\gamma_{s}=0 \mathrm{~dB}$ e $\gamma_{o}=6 \mathrm{~dB}$, realizou-se simulações para verificar o efeito do canal mantendo-se o valor de $\gamma_{0}$ em $6 \mathrm{~dB}$. Os resultados encontram-se na Figura 5, para estas simulações a matriz baseada no $\mathrm{BCH}$ tem um desempenho ligeiramente superior em todo o intervalo analisado.

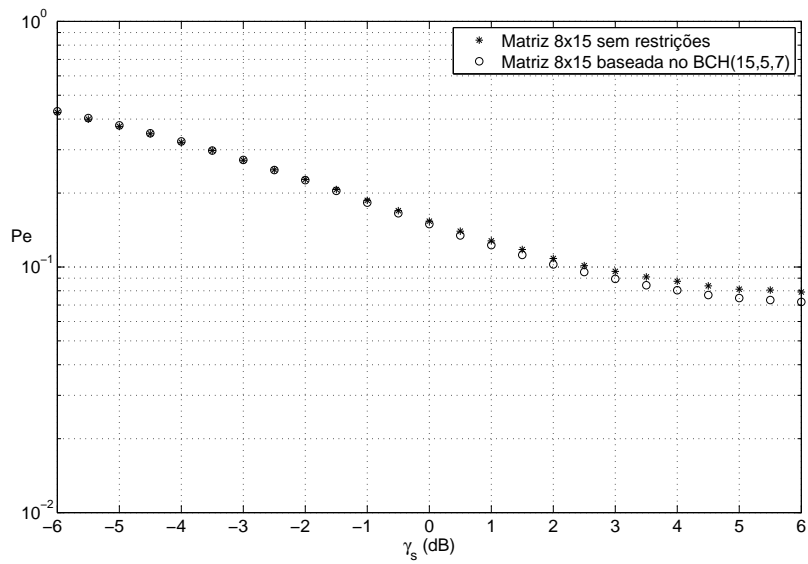

Fig. 5. Simulações para matrizes $8 \times 15$ selecionadas para $\gamma_{s}=0 \mathrm{~dB}$ $\gamma_{o}=6 \mathrm{~dB}$. As simulações foram realizadas para $\gamma_{o}=6 \mathrm{~dB}$.

Também foram feitas avaliações para redes de sensores com uma quantidade maior de nós sensores. Escolheu-se $\gamma_{s}=0 \mathrm{~dB}$ e $\gamma_{o}=6 \mathrm{~dB}$ como razões sinal-ruído alvo durante a seleção das matrizes. Foram avaliados sistemas com $M=8$ e $N=511$ e com $M=16$ e $N=511$. As matrizes foram selecionadas com base no $\operatorname{BCH}(511,10,223)$. Os resultados encontram-se nas Figuras 6 e 7.

Observando os gráficos das Figuras 6 e 7 verifica-se que assim como ocorreu para as redes com uma quantidade pequena de nós sensores, as matrizes códigos baseadas no
$\mathrm{BCH}(511,10,223)$ têm um desempenho tão bom quanto as matrizes obtidas sem nenhuma restrição.

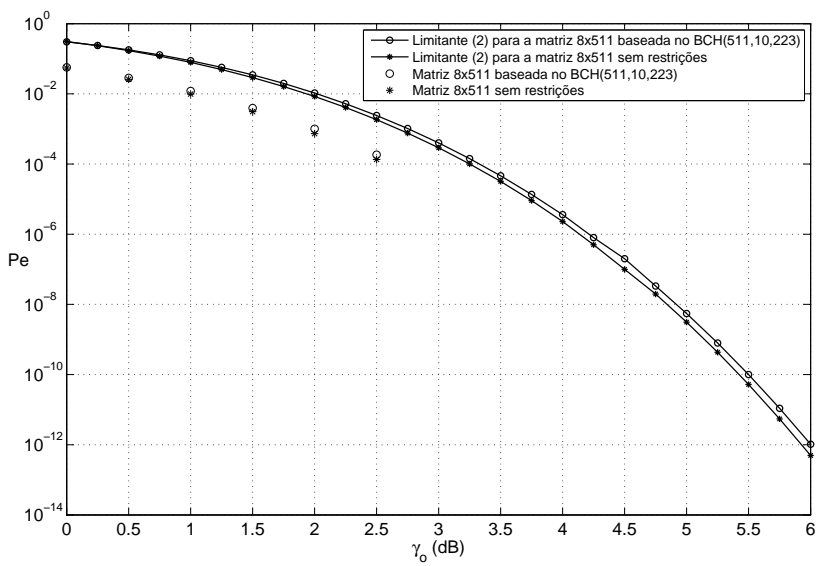

Fig. 6. Simulações e cálculo do limitante (2) para matrizes 8x511 selecionadas para $\gamma_{s}=0 \mathrm{~dB}$ e $\gamma_{o}=6 \mathrm{~dB}$. As simulações foram realizadas para $\gamma_{s}=0 \mathrm{~dB}$.

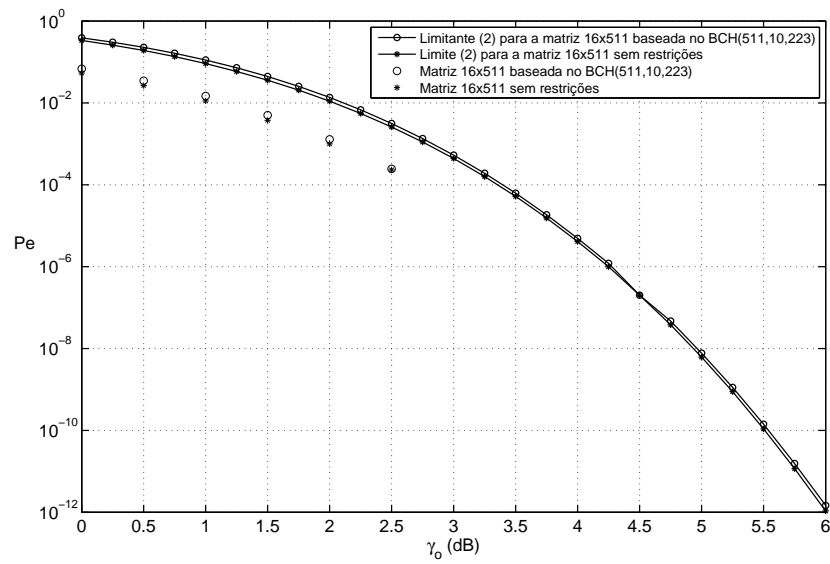

Fig. 7. Simulações e cálculo do limitante (2) para matrizes 16x511 selecionadas para $\gamma_{s}=0 \mathrm{~dB}$ e $\gamma_{o}=6 \mathrm{~dB}$. As simulações foram realizadas para $\gamma_{s}=0 \mathrm{~dB}$.

Embora a abordagem proposta reduza o espaço das possíveis soluções, o novo espaço de busca é mais estruturado e um limitante inferior (distância mínima do código $\mathrm{BCH}$ escolhido) para a distância mínima entre as palavras-código é inicialmente conhecido, não sendo necessário avaliar este parâmetro durante o processo de busca da matriz código.

Em todas as seleções as matrizes com código $\mathrm{BCH}$ obtiveram um desempenho ligeiramente inferior para baixos valores de $\gamma_{o}$ e um desempenho melhor para valores mais altos de $\gamma_{o}$, quando resta apenas o efeito do canal. Uma explicação encontra-se no fato das palavras código da matriz baseada no $\mathrm{BCH}$ possuírem uma distância mínima maior.

Quando as matrizes são selecionadas para um valor baixo de $\gamma_{o}$ pode ser mais interessante que a distância entre palavras vizinhas da matriz (linhas vizinhas) seja pequena, pois quando o nó sensor errar na classificação, tomando como verdadeira uma hipótese adjacente, não ocorrerá erro se o valor binário que ele deve enviar for o mesmo da hipótese verdadeira. Para as matrizes selecionadas com base em um código aleatório 
esse valor da distância não têm um limitante inferior como no caso das matrizes baseadas em um código $\mathrm{BCH}$.

Então quando os valores de $\gamma_{o}$ aumentam, os erros de classificação do nó sensor diminuem e as matrizes baseadas no código $\mathrm{BCH}$ por apresentarem distâncias maiores entre as palavras código passam a ter um desempenho melhor, pois o erro no enlace de comunicação passa a ser mais importante do que erro de classificação do nó sensor.

Por exemplo para o caso de $N=15$ e $M=8$, em que as matrizes foram selecionadas para $\gamma_{s}=0 \mathrm{~dB}$ e $\gamma_{o}=$ $4 \mathrm{~dB}$, e o gráfico das simulações encontra-se na Figura 4, a distância mínima para a matriz baseada em código aleatório é 4 enquanto que para a matriz baseada no código $\mathrm{BCH}$ é 7 , que corresponde a distância mínima do código $\mathrm{BCH}(15,5,7)$.

\section{CONCLUSÕES}

Este trabalho desenvolveu um sistema de classificação distribuída tolerante a falhas baseado no uso de códigos $\mathrm{BCH}$. Nesta abordagem as palavras códigos que formam a matriz código, que representa as regras de decisão nos nós sensores e no centro de fusão, são obtidas de códigos BCH. Propôse também o uso de algoritmo genético para a seleção das melhores matrizes.

Foram feitas simulações para verificar o desempenho das matrizes obtidas por busca em código $\mathrm{BCH}$, e estes resultados foram comparados com o de matrizes selecionadas sem a restrição de suas palavras pertencerem a um determinado código como em [7].

Foi mostrado que não ocorre uma perda significativa de desempenho quando é usada a abordagem aqui proposta. Esta tem a vantagem de um código mais estruturado e a possibilidade de um processo de decodificação menos exaustivo, o que é particularmente interessante se o número de hipóteses for muito grande.

\section{REFERÊNCIAS}

[1] I. Akyildiz, W. Su, Y. Sankarasubramaniam e E. Cayirci. "A survey on sensor networks," Communications Magazine, IEEE, 40(8):102-114, Aug 2002.

[2] H. Wang, J. Elson, L. Girod, D. Estrin e K. Yao. "Target classification and localization in habitat monitoring,"Acoustics, Speech, and Signal Processing, 2003. Proceedings. (ICASSP '03). 2003 IEEE International Conference on, 4:IV-844-7 vol.4, April 2003.

[3] J.-F. Chamberland e V. Veeravalli. "Decentralized detection in sensor networks,"Signal Processing, IEEE Transactions on, 51(2):407-416, Feb 2003.

[4] S. Aldosari e J. Moura. "Detection in decentralized sensor networks,"Acoustics, Speech, and Signal Processing, 2004. Proceedings. (ICASSP'04). IEEE International Conference on, 2:ii-277-80 vol.2, May 2004.

[5] G. J. Pottie e W. J. Kaiser. "Wireless integrated network sensors" Commun. ACM, 43(5):51-58, 2000.

[6] T.-Y. Wang, Y. Han, P. Varshney, and P.-N. Chen. "Distributed fault tolerant classification in wireless sensor networks". Selected Areas in Communications, IEEE Journal on, 23(4):724-734, April 2005.

[7] C. Yao, P.-N. Chen, T.-Y. Wang, Y. S. Han, e P. K. Varshney. "Performance analysis and code design for minimum hamming distance fusion in wireless sensor networks". Information Theory, IEEE Transactions on,53(5):1716-1734, May 2007.

[8] F. M. de Assis. "Genetic algorithms and packing of block codes". International Conference on Tele-comunications, Proceedings of the ICT97, 3:1045-1048, 1997.

[9] F. M. de Assis. "Weight structure of binary codes and the performance of blind search algorithms". Neural Networks, Brazilian Symposium on, $0: 144,2000$
[10] P.-N. Chen, T.-Y. Wang, Y. Han, P. Varshney e C. Yao. "Asymptotic performance analysis for minimum hamming distance fusion,"Acoustics, Speech, and Signal Processing, 2005. Proceedings. (ICASSP '05). IEEE International Conference on, 4:iv/865-iv/868 Vol. 4, March 2005. 